\title{
TRAVELLING WAVE SOLUTIONS TO A GRADIENT SYSTEM
}

\author{
JAMES F. REINECK
}

\begin{abstract}
Given a system of reaction-diffusion equations where the nonlinearity is derived from a potential with certain restrictions, we use the Conley index and the connection matrix to show that there is a travelling wave solution connecting the maxima of the potential.
\end{abstract}

1. Introduction. We consider a system of reaction-diffusion equations

$$
\frac{\partial u_{i}}{\partial \tau}=\frac{\partial^{2} u}{\partial \xi^{2}}+f_{i}\left(u_{1}, \ldots, u_{d}\right), \quad i=1, \ldots, d,
$$

where $u(\xi, \tau): \mathbf{R}^{2} \rightarrow \mathbf{R}$. We assume that the $f_{i}$ are derived from a potential $F$, i.e.

$$
f_{i}\left(u_{1}, \ldots, u_{d}\right)=F_{u_{i}}\left(u_{1}, \ldots, u_{d}\right), \quad i=1, \ldots, d,
$$

for some $F: \mathbf{R}^{d} \rightarrow \mathbf{R}$. We are looking for travelling wave solutions of 1.1, i.e. nonconstant, bounded solutions of the form

$$
u_{i}(\xi, \tau)=u_{i}(\xi+\theta \tau), \quad i=1, \ldots, d .
$$

Thus, each $u_{i}$ is a function of $\xi+\theta \tau$ alone. We write $u_{i}(t)$ for $u_{i}(\xi+\theta \tau)$. The system 1.1 then becomes a system of ordinary differential equations

$$
u_{i}^{\prime \prime}=\theta u_{i}^{\prime}-f_{i}\left(u_{1}, \ldots, u_{d}\right), \quad i=1, \ldots, d,
$$

where ' denotes $d / d t$. We can write this as a system of first order equations. We introduce the notation $U(t)=\left(u_{1}(t), \ldots, u_{d}(t)\right), V(t)=\left(v_{1}(t), \ldots, v_{d}(t)\right)$. The system then becomes

$$
\begin{aligned}
& U^{\prime}=V, \\
& V^{\prime}=\theta V-\nabla F(U) .
\end{aligned}
$$

We assume that over some convex set $N_{1}, F$ has three critical points, two maxima $M_{1}$ and $M_{2}$, and a point $M_{3}$ of Morse index $d-1$. (Precise assumptions on $F$ will be given below.) We will be interested in solutions which satisfy $\theta>0$ and

$$
U(t) \rightarrow M_{1} \quad \text { as } t \rightarrow+\infty, \quad U(t) \rightarrow M_{2} \quad \text { as } t \rightarrow-\infty,
$$

i.e. the solution $(U(t), V(t))$ is a connecting orbit between the rest points $\left(M_{2}, 0\right)$ and $\left(M_{1}, 0\right)$ in the local flow generated by 1.2 .

Let $H(U, V)=\|V\|^{2} / 2+F(U) . H: \mathbf{R}^{d} \times \mathbf{R}^{d} \rightarrow \mathbf{R}$ is called the energy function. Along any solution $(U(t), V(t))$, we have $(d / d t) H(U(t), V(t))=\theta\|V\|^{2}$. Thus for $\theta \neq 0, H$ is a Lyaponov function and the flow generated by 1.2 is gradientlike. It

Received by the editors September 18, 1986 and, in revised form, February 9, 1987.

1980 Mathematics Subject Classification (1985 Revision). Primary 35K55; Secondary 58E99.

Supported in part by the National Science Foundation under grant \#0830-300-L655. 
follows that the only bounded solutions are critical points and orbits connecting the critical points. In contrast, when $\theta=0$ we have a Hamiltonian system.

We make the following assumptions on $F$ :

A1. $F \in C^{2}\left(\mathbf{R}^{d}, \mathbf{R}\right)$.

A2. There is a compact, convex set $N_{1} \subset \mathbf{R}^{d}$ such that $N_{1}$ is a component of $F^{-1}([\alpha, \infty))$ for some number $\alpha$.

A3. The restriction of $F$ to $N_{1}$ has three nondegenerate critical points, two maxima $M_{1}$ and $M_{2}$, and a point $M_{3}$ of Morse index $d-1 . F\left(M_{3}\right)<F\left(M_{2}\right)<$ $F\left(M_{1}\right)$.

A4. $\left(2 F\left(M_{1}\right)-2 F(U)-\left\langle U-M_{1}, \nabla F(U)\right\rangle\right) \geq 0$ for all $(U, V) \in\left[W^{u}\left(M_{1}, 0\right) \cup\right.$ $\left.W^{s}\left(M_{1}, 0\right)\right]$ in the flow from 1.2 with $\theta=0$.

REMARKS 1.4. The convexity of $N_{1}$ will be used to construct an isolating neighborhood in $\mathbf{R}^{d} \times \mathbf{R}^{d}$. A3 can be weakened somewhat. $M_{3}$ need not be a single rest point, but can be replaced by a collection of several rest points and orbits connecting them. This will be discussed in $\S 3$.

Hypothesis A4 can also be weakened. The precise hypothesis is

$\mathrm{A} 4^{\prime}$. In the flow generated by 1.2 with $\theta=0$, there is no connected collection of bounded orbits containing the rest points $\left(M_{1}, 0\right)$ and $\left(M_{2}, 0\right)$.

Some assumption such as A4 or A4' is needed to prevent "pathologies", i.e., the very strange behavior which has been observed in Hamiltonian systems. A4 is easier to verify, so we state the main result using it. The inequality in A4 may hold for all $U$ in $N_{1}$, for example.

The main result is

THEOREM 1.5. Let $F$ satisfy A1-A4. Then there is a solution of 1.2, 1.3 for some $\theta>0$.

In [1] Conley sketches a proof of Theorem 1.5 using the continuation property of the Conley index. The proof we give here illustrates the use of the connection matrix and avoids some difficult estimates in the case $d \geq 2$.

In $\S 2$ we construct an isolating neighborhood for the system. In $\S 3$ we use the Conley index and connection matrix to prove Theorem 1.5 under the assumptions A1-A3, A4 $4^{\prime}$. In $\S 4$ we show that A4 implies A4'.

ACKNOWLEDGMENT. I would like to thank David Terman for suggesting the problem and for helpful discussions.

2. An isolating neighborhood. To apply the Conley index and connection matrix to our problem, we need to construct an isolating neighborhood in $\mathbf{R}^{d} \times \mathbf{R}^{d}$ for an interval of $\theta$ values. Also, we will deform the flow to simplify computations. We begin by defining two deformations. Let $G_{1}: \mathbf{R}^{d} \rightarrow \mathbf{R}^{d}$ have the properties

(a) $G_{1}(x)=F(x)$ in a neighborhood of $M_{1}$.

(b) $G_{1}(x)=F(x)$ for all $x \notin \operatorname{int}\left(N_{1}\right)$.

(c) $M_{1}$ is the only critical point of $G_{1}$ in $N_{1}$.

Such a $G_{1}$ exists because $M_{1}$ is a maximum and $N_{1}$ is convex. $G_{2}$ is defined similarly, with $M_{2}$ replacing $M_{1}$ in (a) and (c). For $i=1,2$, define homotopies

$$
H_{i}(x, s)=s G_{i}(x)+(1-s) F(x), \quad s \in[0,1] .
$$

Note that for each $s$, the function $H_{i}(\cdot, s)$ still satisfies assumption A2. 
For $i=1,2, s \in[0,1]$, we have equations

$$
U^{\prime}=V, \quad V^{\prime}=\theta V-\nabla H_{i}(U, s) .
$$

where $\nabla H_{i}(U, s)$ means gradient in the $u_{i}$ only.

LEMMA 2.1. Suppose $(U(t), V(t))$ is a solution to 1.2.i.s with $U\left(t_{0}\right) \in \partial N_{1}$. Then $U\left(t_{0}\right)$ is not internally tangent to $N_{1}$, i.e. for any $\varepsilon>0$, there is a $t$ with $\left|t-t_{0}\right|<\varepsilon$ and $U(t) \notin N_{1}$.

PROOF. The proof follows Conley [1].

$$
\left.\frac{d}{d t} H_{i}(U(t), s)\right|_{t_{0}}=\left\langle\nabla H_{i}\left(U\left(t_{0}\right), s\right), V\left(t_{0}\right)\right\rangle .
$$

If this is not 0 , then $U(t)$ crosses $\partial N_{1}$ transversally, and the result is clear. So suppose $\left.(d / d t) H_{i}(U(t), s)\right|_{t_{0}}=0$. Then

$$
\left.\frac{d^{2}}{d t^{2}} H_{i}(U(t), s)\right|_{t_{0}}=d^{2} F\left(U\left(t_{0}\right)\right)+\theta\left\langle\nabla H_{i}\left(U\left(t_{0}\right), s\right), V\left(t_{0}\right)\right\rangle-\left\|\nabla H_{i}\left(U\left(t_{0}\right), s\right)\right\|^{2}<0
$$

since $N_{1}$ is convex.

LEMMA 2.2. There is a $T>0$ such that for any $s \in[0,1], \theta \geq T$ there is no solution $(U(t), V(t))$ of 1.2.2.s with $U(t) \in N_{1}$ for all $t$ and $U(t) \rightarrow M_{2}$ as $t \rightarrow-\infty$. Similarly, if $\theta \leq-T$, then there is no solution with $U(t) \in N_{1}$ for all $t$ and $U(t) \rightarrow M_{2}$ as $t \rightarrow+\infty$.

Proof. This proof is due to Terman [7]. We do the case $\theta>0$; a similar argument works for $\theta<0$.

For $\lambda>0$, let

$$
S_{\lambda}=\left\{(U, V)|| V_{i}|\geq \lambda| U_{i}-\left(M_{2}\right)_{i} \mid\right\}
$$

where $\left(M_{2}\right)_{i}$ denotes the $i$ th coordinate of the rest point $M_{2} \in \mathbf{R}^{d}$. We show that there is a $T_{\lambda}>0$ such that $\theta>T_{\lambda}$ implies that $S_{\lambda}$ is positively invariant relative to $N_{1} \times \mathbf{R}^{d}$. We do this by computing the vector field on $\partial S_{\lambda}$ and show that it points into $S_{\lambda}$. A boundary face of $S_{\lambda}$ has the form

$$
\left|V_{i}\right|=\lambda\left|U_{i}-\left(M_{2}\right)_{i}\right| \quad \text { for some } i, \quad\left|V_{k}\right| \geq \lambda\left|U_{k}-\left(M_{2}\right)_{k}\right| \quad \text { for } k \neq i .
$$

There are four cases, e.g., $V_{i}=\lambda\left(U_{i}-\left(M_{2}\right)_{i}\right), V_{i}>0$. Let $\left(u_{i}, v_{i}\right)=(\lambda,-1)$ be the outward normal. Then

$$
\begin{aligned}
(\lambda,-1) \cdot\left(u_{i}^{\prime}, v_{i}^{\prime}\right) & =\lambda v_{i}-\theta v_{i}+\partial_{u_{i}} H_{2}(U, s) \\
& =(\lambda-\theta)\left(U_{i}-\left(M_{2}\right)_{i}\right)+\partial_{u_{i}} H_{2}(U, s) \\
& <0
\end{aligned}
$$

if $\theta>0$ is large because $M_{2}$ is a maximum of $F$, hence of $H_{2}(\cdot, s)$. The other cases are similar.

To complete the proof, we compute the eigenvectors of the linearized equation at $M_{2}$ and show that they point into $S_{\lambda}$ if $\theta$ is large enough. Since $S_{\lambda}$ contains no rest points, this will establish the result. Let $M=$ Hessian of $F$ at $M_{2}$. Since $M_{2}$ is a local maximum, the eigenvalues of $M$ at $M_{2}$ are all negative, $-\lambda_{1}, \ldots,-\lambda_{d}$ 
with eigenvectors $w_{1}, \ldots, w_{d}$. The matrix for the linearized system is $\left[\begin{array}{cc}0 & I \\ -M & \theta I\end{array}\right]$. The characteristic polynomial (in $x$ ) is

$$
\prod_{i=1}^{d}\left(x(x-\theta)-\lambda_{i}\right)
$$

so the eigenvalues are

$$
x^{ \pm}=\theta \pm \sqrt{\theta^{2}+4 \lambda_{i}} .
$$

The eigenvector associated to $x_{i}$ is $\left(w_{i}, x_{i} w_{i}\right) . x_{i} \rightarrow \infty$ as $\theta \rightarrow \infty$ so $x_{i}$ points into $S_{\lambda}$ for $\theta$ sufficiently large.

For an isolated invariant set $S$, let $h(S)$ denote its Conley index (see $[\mathbf{1}, \mathbf{6}]$ ). $\Sigma^{k}$ denotes the pointed $k$-sphere.

LEMMA 2.4. For $\theta>0, h\left(\left(M_{1}, 0\right)\right)=h\left(\left(M_{2}, 0\right)\right)=\Sigma^{d}, h\left(\left(M_{3}, 0\right)\right)=\Sigma^{d+1}$.

For $\theta<0, h\left(\left(M_{1}, 0\right)\right)=h\left(\left(M_{2}, 0\right)\right)=\Sigma^{d}, h\left(\left(M_{3}, 0\right)\right)=\Sigma^{d-1}$.

PROOF. For a nondegenerate rest point $p, h(p)=\Sigma^{k}$ where $k$ is the number of eigenvalues of the linearized equation with positive real part. The result now follows easily from the form of the characteristic polynomial 2.3 .

The following lemma is also from Terman's paper [7].

LEMMA 2.5. Let $T$ be as in Lemma 2.2. Then there is a number $K$ such that $U\left(t_{0}\right) \in N_{1}$ and $\left\|V\left(t_{0}\right)\right\|>K$ implies $\{U(t) \mid t \in \mathbf{R}\} \not \subset N_{1}$ in the flow generated by 1.2.i.s for $i=1,2, s \in[0,1]$ and $|\theta| \leq T$. $K$ depends on $T$, a bound on $\nabla H_{i}(U, s)$ for $U \in N_{1}$, and the diameter of $N_{1}$.

Let $N=N_{1} \times\left\{V \mid\left\|V\left(t_{0}\right)\right\|<K\right\}$. From Lemmas 2.1 and 2.5 it follows that $N$ is an isolating neighborhood for the flow generated by 1.2 with $|\theta| \leq T$, hence for $|\theta| \leq T+\delta$ for sufficiently small $\delta$. For any such $\theta$, let $S_{\theta}$ be the maximal invariant set contained in $N$. Since $H$ is a Lyaponov function for $\theta \neq 0$, it follows that the critical points $\left\{\left(M_{i}, 0\right) \mid i=1,2,3\right\}$ form a Morse decomposition of $S_{\theta}$ for $\theta \neq 0$.

3. The existence of a connection. Our plan to show the existence of a solution to $1.2,1.3$ with positive $\theta$ is as follows. We consider a flow on $\mathbf{R}^{d} \times \mathbf{R}^{d} \times \mathbf{R}$ with coordinates $(U, V, \theta)$. The critical points of this flow will be $\left(M_{i}, 0, T\right)$ and $\left(M_{i}, 0,-T\right), i=1,2,3$. We will show that for any small drift in the $-\theta$ direction, there is a connection from $\left(M_{2}, 0, T\right)$ to $\left(M_{1}, 0,-T\right)$. We let the drift go to zero and obtain a set which is the limit of these connections. This limit contains the solution to $1.2,1.3$.

We assume that the reader is familiar with Morse decompositions, the Conley index, and index filtrations. References include $[\mathbf{1}, \mathbf{2}, \mathbf{3}$, and 6].

Notation. Let $\left\{M_{\pi} \mid \pi \in P\right\}$ be a Morse decomposition of $S$ with $P$ a partially ordered set. Choose an index filtration. Let $I \subset P$ be an interval. Then

$$
\begin{gathered}
C\left(M_{\pi}, M_{\pi^{\prime}}\right)=\left\{x \mid \omega^{*}(x) \subset M_{\pi}, \omega(x) \subset M_{\pi^{\prime}}\right\}, \\
M(I)=\left(\bigcup_{\pi \in P} M_{\pi}\right) \cup\left(\bigcup_{\pi, \pi^{\prime} \in P} C\left(M_{\pi}, M_{\pi^{\prime}}\right)\right), \\
H(\pi)=H_{*}\left(h\left(M_{i}\right) ; \mathbf{Z}_{2}\right) \quad(\text { singular homology) } \\
H(I)=H_{*}\left(h(M(I)) ; \mathbf{Z}_{2}\right) \quad \text { (singular homology). }
\end{gathered}
$$


We briefly outline the portions of the connection matrix theory which we shall use. Details can be found in [4]. If $\left(A^{*}, A\right)$ is an attractor-repeller pair in an isolated invariant set $S$, then we can find a compact triple of spaces $\left(N_{2}, N_{1}, N_{0}\right)$ such that $\left(N_{2}, N_{0}\right)$ is an index pair for $S,\left(N_{1}, N_{0}\right)$ is an index pair for $A$, and $\left(N_{2}, N_{1}\right)$ is an index pair for $A^{*}$. We consider $N_{2} / N_{1}$ as a pointed space with the equivalence class of $N_{1}$ as the distinguished point, and similarly for the other two pairs. There is a long exact homology sequence of pointed spaces

$$
\cdots \rightarrow H_{q}\left(N_{1} / N_{0}\right) \rightarrow H_{q}\left(N_{2} / N_{0}\right) \rightarrow H_{q}\left(N_{2} / N_{1}\right) \stackrel{\partial}{\rightarrow} H_{q-1}\left(N_{1} / N_{0}\right) \rightarrow \cdots
$$

Since this is essentially independent of the triple, we write

$$
\cdots \rightarrow H_{q}(A) \rightarrow H_{q}(S) \rightarrow H_{q}\left(A^{*}\right) \stackrel{\partial}{\rightarrow} H_{q-1}(A) \rightarrow \cdots
$$

where $H_{q}(S)$ denotes $H_{q}(h(S))$, etc. We call $\partial$ the flow defined boundary map. Exactness implies that if $H(S)=0$, then $\partial$ is an isomorphism. If $C\left(A^{*}, A\right) \neq \varnothing$, then $H(S)=H(A) \oplus H\left(A^{*}\right)$, and it follows that $\partial$ is identically zero. So we have

LEMMA 3.1. If $\partial \neq 0$, then $C\left(A^{*}, A\right) \neq \varnothing$.

Given a Morse decomposition $\left\{M_{\pi} \mid \pi \in P\right\}$, if $\pi$ and $\pi^{\prime}$ are adjacent in the partial order with $\pi<\pi^{\prime}$, then $\left(M(\pi), M\left(\pi^{\prime}\right)\right)$ forms an attractor-repreller pair in $M\left(\pi, \pi^{\prime}\right)$. It follows that there is a flow defined boundary map $\partial\left(\pi^{\prime}, \pi\right)$. The connection matrix puts restrictions on these maps, and allows us to compute unknown maps from known ones. For an interval $I$ in $P$, we define

$$
C \Delta(I)=\bigoplus_{\pi \in I} H(\pi)
$$

A map $\Delta(I): C \Delta(I) \rightarrow C \Delta(I)$ can be thought of as a matrix $\left[\Delta\left(\pi^{\prime}, \pi\right): H\left(\pi^{\prime}\right) \rightarrow\right.$ $H(\pi)$ ] indexed by pairs of elements of $I . \Delta(P)$ is called upper triangular if $\Delta\left(\pi^{\prime}, \pi\right)$ $=0$ if $\pi \nless \pi^{\prime} . \Delta(P)$ is called a boundary map if each $\Delta\left(\pi^{\prime}, \pi\right)$ has degree -1 and $(\Delta(P))^{2}=0$. It is not difficult to show that if $\Delta(P)$ is an upper triangular boundary map, then so is the restriction $\Delta(I)$ to any interval $I$. Thus $(C \Delta(I), \Delta(I))$ is a chain complex. Let $H \Delta(I)$ denote its homology. An upper triangular boundary map $\Delta(P)$ is called a connection matrix if

(i) for $\pi<\pi^{\prime}$ and $\pi$ and $\pi^{\prime}$ adjacent, $\Delta\left(\pi^{\prime}, \pi\right): H\left(\pi^{\prime}\right) \rightarrow H(\pi)$ is the flow defined boundary map.

(ii) $H \Delta(I)$ is isomorphic to $H(I)$ for any interval $I$.

The existence of connection matrices is shown by Franzosa in [4]. The brief description here is incomplete, but it suffices for our application. The $\Delta^{2}=0$ condition allows one to conclude that certain maps are nonzero. Condition (ii) is essentially a rank condition. Since the homologies are $\mathbf{Z}_{2}$ vector spaces we have

$$
\operatorname{dim} H(I)=\operatorname{dim} H \Delta(I)=\operatorname{dim}(\operatorname{ker} \Delta(I))-\operatorname{rank} \Delta(I) .
$$

Thus there are restrictions on the rank of $\Delta$ and some submatrices.

LEMMA 3.2. Let $\left\{M_{1}, M_{2}, M_{3}\right\}$ be a three set Morse decomposition of an isolated invariant set $S, M_{2}<M_{1}$ in the flow defined partial order. Suppose that $S$ can be continued to $M_{3}$ and that $M_{3}$ remains a repeller [or attractor] during the 
deformation. Then the flow defined boundary map $\partial(2,1): H_{q}(2) \rightarrow H_{q-1}(1)$ is an isomorphism for all $q$.

ProOF. $(M(1,2), M(3))$ is an attractor-repeller pair. Because $M(3)$ remains a repeller, $M(1,2)$ continues to the empty set, so $H(1,2)=0$, and the map $\partial(1,2)$ is flow defined. The result now follows from the exact sequence

$$
\cdots \rightarrow H_{q}(1) \rightarrow H_{q}(12) \rightarrow H_{q}(2) \stackrel{\partial}{\rightarrow} H_{q-1}(1) \rightarrow \cdots
$$

of the attractor-repeller pair $\left(M_{1}, M_{2}\right)$.

LEMMA 3.3. Let $S$ be the set isolated by $N$ in the flow generated by 1.2 , $\left\{\left(M_{i}, 0\right) \mid i=1,2,3\right\}$ the Morse decomposition for $\theta \neq 0$. Then for $\theta \geq T$, the connection matrix in the flow defined partial order is

$$
\begin{aligned}
& 1 \\
& 2
\end{aligned}\left[\begin{array}{lll}
1 & 2 & 3 \\
0 & 0 & 1 \\
0 & 0 & 1 \\
0 & 0 & 0
\end{array}\right] \text { and for } \theta \leq-T \text { the matrix is } \begin{aligned}
& 1 \\
& 2
\end{aligned}\left[\begin{array}{lll}
1 & 2 & 3 \\
0 & 0 & 0 \\
0 & 0 & 0 \\
0 & 1 & 1
\end{array}\right]
$$

ProOF. For $\theta \geq T$, we can use $H_{1}$ and $H_{2}$ to continue $S$ to $\left(M_{1}, 0\right)$ and $\left(M_{2}, 0\right)$ in such a ways that the critical points remain repellers. For $M_{1}$ this is just the fact that energy increases; for $M_{2}$ it follows from Lemma 2.2. Similarly, for $\theta \leq-T$, we continue $S$ to the critical points in such a way that the critical points remain attractors. Thus the maps $\partial(3,1)$ and $\partial(3,2)$ are isomorphisms for $\theta \geq T$ and the maps $\partial(1,3)$ and $\partial(2,3)$ are isomorphisms for $\theta \leq-T$ by Lemma 3.2. Since $H(1)$ and $H(2)$ are both nonzero in the same dimension, there is no possible nonzero map of degree -1 . The other connection matrix entries are zero by upper triangularity.

We now consider the system on $\mathbf{R}^{d} \times \mathbf{R}^{d} \times \mathbf{R}$

$$
\begin{aligned}
U^{\prime} & =V, \\
V^{\prime} & =\theta V-\nabla F(U), \\
\theta^{\prime} & =\varepsilon(\theta-T)(\theta+T) .
\end{aligned}
$$

The following facts are proved in [5].

LEMMA 3.5. $N \times[-T-\eta, T+\eta], \eta$ small, is an isolating neighborhood for the flow generated by 3.4 if $\varepsilon$ is small enough.

Let $\hat{S}$ denote the set isolated by $N \times[-T-\eta, T+\eta]$.

LEMMA 3.6. $\left\{\left(M_{i}, 0, T\right),\left(M_{i}, 0,-T\right) \mid i=1,2,3\right\}$ forms a Morse decomposition of $\hat{S}$.

LEMMA 3.7. Let $\Delta$ denote the connection matrix for $\hat{S}$. Then $\Delta$ has rank 3 and $\Delta$ has the form (where $1^{-}$denotes $\left(M_{1}, 0,-T\right)$, etc.) :

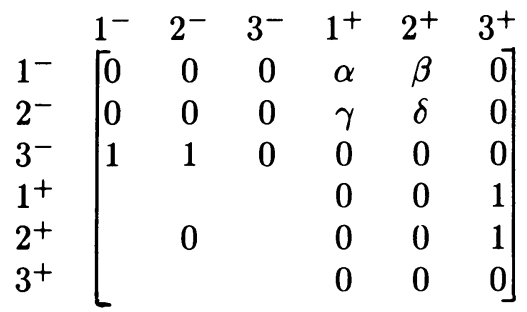

where $\alpha, \beta, \gamma$, and $\delta$ are to be determined. 
The reason for Lemma 3.7 is as follows. $-T$ is a fixed point in the $\theta$ flow with Conley index $\Sigma^{0}$. By a variation of the product theorem for the index, it follows that the rest points $1^{-}, 2^{-}$, and $3^{-}$have the same Conley index in the flow generated by 3.4 as they do in the flow from 1.2. The upper left block of the connection matrix comes from the $\theta=-T$ flow. The index of the fixed point $T$ in the $\theta$ flow is $\Sigma^{1}$ so by the product theorem the indices of $1^{+}, 2^{+}$, and $3^{+}$are spheres of dimension $d+1, d+1$, and $d+2$ respectively. The lower right block of the connection matrix comes from the $\theta=T$ flow. The unknown entries in the upper right block of $\Delta$ represent the connections which drift across from $\theta=T$ to $\theta=-T$. The possible maps of degree -1 are $\alpha, \beta, \gamma$, and $\delta$. The other maps cannot be nontrivial maps of degree -1 . The rank condition on $\Delta$ is seen as follows. The Conley index of $\hat{S}$ is 0 . This can be seen by "cancelling" the rest points at $\theta=T$ and $\theta=-T$ so $\hat{S}$ continues to the empty set. Thus

$$
\operatorname{dim}(\operatorname{ker} \Delta)-\operatorname{rank} \Delta=0, \quad \operatorname{dim}(\operatorname{ker} \Delta)+\operatorname{rank} \Delta=6
$$

so $\operatorname{rank} \Delta=3$.

Since $\Delta^{2}=0$, it follows that $\alpha=\beta=\gamma=\delta$. The rank condition implies that the common value is 1 . Thus $\left(M_{1}, 0,-T\right)$ lies below $\left(M_{2}, 0, T\right)$ in the flow defined partial order. It follows that there is a finite sequence of Morse sets starting at $\left(M_{2}, 0, T\right)$ and ending at $\left(M_{1}, 0,-T\right)$ with a connection existing between adjacent sets in the sequence. By Lemma 2.2, $\left(M_{2}, 0, T\right)$ is an attractor in the $\theta=T$ flow, so the second set in the sequence must be $\left(M_{i}, 0,-T\right)$ for some $i$. If $i$ is not 1 , then the sequence can never reach $\left(M_{1}, 0,-T\right)$ since it is a repeller. Thus there is an orbit connecting $\left(M_{2}, 0, T\right)$ and $\left(M_{1}, 0,-T\right)$ for any small $\varepsilon$. Let $\varepsilon_{n}$ be a sequence of positive numbers tending to 0 . For each $n$ we get a connecting orbit $c_{n}$ from $\left(M_{2}, 0, T\right)$ to $\left(M_{1}, 0,-T\right)$ in the flow generated by 3.4. The set $\operatorname{cl}\left(c_{n}\right)=c_{n} \cup\left\{\left(M_{2}, 0, T\right),\left(M_{1}, 0,-T\right)\right\}$ is compact, so the sequence $\operatorname{cl}\left(c_{n}\right)$ has a convergent subsequence on the Hausdorff metric on the compact subsets of $N \times[-T-\eta, T+\eta]$. Denote this limit by $c$, and let $c(\theta)=c \cap\left(\mathbf{R}^{d} \times \mathbf{R}^{d} \times\{\theta\}\right)$.

LEMMA 3.8. (See [5].) $c$ is compact and connected. For $\theta \in[-T, T] c(\theta)$ is nonempty, compact, connected and invariant in the flow generated by 1.2. For $\theta \neq 0$, the set $\left\{i \mid c(\theta) \cap\left(M_{i}, 0, \theta\right) \neq \varnothing\right\}$ is a totally ordered subset in the flow defined partial order on $\left\{\left(M_{i}, 0, \theta\right) \mid i=1,2,3\right\}$.

The fact that $c(\theta)$ is a compact invariant subset of $N$ implies that it consists of rest points and connecting orbits.

For $i=1,2,3$ define $B_{i}=\left\{\theta \mid c(\theta) \subset\left(M_{i}, 0, \theta\right)\right\}$.

LEMMA 3.9. (See [5].) $B_{i}$ is open in $[-T, 0) \cup(0, T]$. For $\theta \neq 0$, let

$$
\begin{aligned}
& j=\inf \left\{j \mid c(\theta) \cap\left(M_{j}, 0, \theta\right) \neq \varnothing\right\}, \\
& k=\sup \left\{k \mid c(\theta) \cap\left(M_{k}, 0, \theta\right) \neq \varnothing\right\}
\end{aligned}
$$

(the inf and sup in the flow defined order). Then there is a $\delta>0$ such that $(\theta-\delta, \theta) \subset B_{j}$ and $(\theta, \theta+\delta) \subset B_{k}$.

Thus $c$ consists of pieces of Morse set and discrete jumps. Because the Morse decomposition breaks down at $\theta=0$, these jumps could, in theory, cluster at $\theta=0$. However, the energy function prevents this. 
We can now establish the existence of a travelling wave solution for the system with hypothesis $\mathrm{A} 4^{\prime}$.

THEOREM 3.10. Let $F$ satisfy A1-A3, A4'. Then there is a $\theta \in(0, T)$ such that there is a connection from $\left(M_{2}, 0\right)$ to $\left(M_{1}, 0\right)$ in the flow generated by 1.2.

ProOf. $-T \in B_{1}$. Let $\underline{\theta}=\sup \left\{\theta \mid[-T, \theta] \subset B_{1}\right\}$. Since $\left(M_{1}, 0\right)$ is a repeller for $\theta<0$, Lemma 3.8 implies $\underline{\theta} \geq 0$. Since $T \in B_{2}, \underline{\theta}<T$.

Case 1. $\underline{\theta}>0$. Then there is a connection from $\left(M_{2}, 0\right)$ to $\left(M_{1}, 0\right)$ at $\underline{\theta}$. If not, then $c(\underline{\theta}) \cap\left(M_{3}, 0, \underline{\theta}\right) \neq \varnothing . M_{3}$ is a repeller in $S_{\theta}$ for $\theta>0$ because energy increases, so Lemma 3.9 would imply $(\underline{\theta}, T) \subset B_{3}$ which is impossible.

Case 2. $\underline{\theta}=0$. Then define $\bar{\theta}=\inf \left\{\theta \mid[\theta, T] \subset B_{2}\right\} . \bar{\theta} \geq 0$ since $\underline{\theta}=0$. If $\bar{\theta}>0$, then the fact that $M_{3}$ is a repeller implies $c(\bar{\theta}) \cap\left(M_{1}, 0, \bar{\theta}\right) \neq \varnothing$. So we have a connection at $\bar{\theta}$. We finish the proof by showing that $\bar{\theta}=0$ is impossible.

Suppose $\bar{\theta}=0$. Since $c$ is compact, both $\left(M_{1}, 0\right)$ and $\left(M_{2}, 0\right)$ are contained in $c(0) . c(0)$ is compact and invariant, so it consists of bounded orbits. By Lemma $3.5, c(0)$ is connected. Thus $c(0)$ is a connected set of bounded orbits in the flow for $\theta=0$ which contains $\left(M_{1}, 0\right)$ and $\left(M_{2}, 0\right)$, contradicting $\mathrm{A} 4^{\prime}$.

REMARK 3.11. The above argument does not require $\left(M_{3}, 0\right)$ to be a critical point. $M_{3}$ could consist of several critical points and connecting orbits, and $M_{3}$ could vary with $\theta$ as connections come and go. What is needed is

(a) $h\left(\left(M_{3}, 0\right)\right)=\Sigma^{d-1}$ for $\theta=-T, h\left(\left(M_{3}, 0\right)\right)=\Sigma^{d+1}$ for $\theta=+T$.

(b) $\left\{\left(M_{1}, 0\right),\left(M_{2}, 0\right),\left(M_{3}, 0\right)\right\}$ for a Morse decomposition of $S_{\theta}$ for $\theta \neq 0$.

(c) For $\theta= \pm T$, the connection matrices are as in Lemma 3.3, i.e. we can deform as in Lemma 3.2 .

(d) $F(u)<F\left(M_{2}\right)$ for any $u \in M_{3}$.

Thus A3 can be weakened considerably. A picture of $F$ for $d=2$ is shown in Figure 1. $M_{3}$ consists of several critical points and connections.

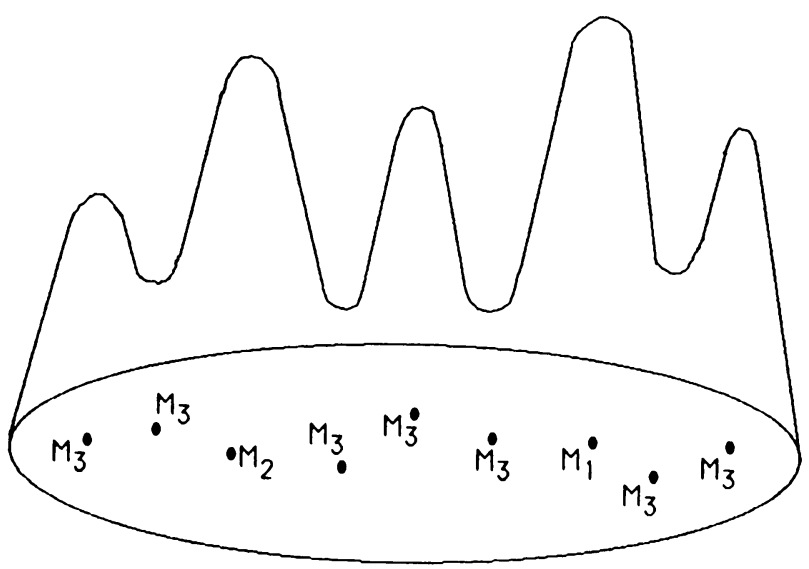

FIGURE 1 
4. Separation of critical points. In this section we show that the inequality A4 implies $\mathrm{A}^{\prime}$.

LEMMA 4.1. Assume A4. Let

$$
(U(0), V(0)) \in\left[W^{u}\left(M_{1}, 0\right) \cup W^{s}\left(M_{1}, 0\right)\right] \backslash\left\{\left(M_{1}, 0\right)\right\}
$$

for the flow from 1.2 with $\theta=0$. Then there is a $t \in \mathbf{R}$ with $U(t) \notin N_{1}$.

Proof. We may assume $M_{1}=0$. Then $\|U\|$ is the distance from $U$ to $M_{1}$. We have $d\|U\|^{2} / d t=2\langle U, V\rangle$. This is positive for $(U, V) \in W^{u}\left(M_{1}, 0\right) \backslash\left\{\left(M_{1}, 0\right)\right\}$, negative for $(U, V) \in W^{s}\left(M_{1}, 0\right) \backslash\left\{\left(M_{1}, 0\right)\right\}$.

$$
\frac{d^{2}}{d t^{2}}\|U\|^{2}=2(\langle V, V\rangle-\langle U, \nabla F(U)\rangle)=2\left(2 F\left(M_{1}\right)-2 F(U)-\langle U, \nabla F(U)\rangle\right)
$$

since the stable and unstable manifolds are contained in the energy level set $H^{-1}\left(F\left(M_{1}\right)\right)$. Since this is nonnegative, it follows that $d\|U\|^{2} / d t$ is larger than a positive constant in forward time for $(U, V) \in W^{u}\left(M_{1}, 0\right) \backslash\left\{\left(M_{1}, 0\right)\right\}$ and in backward time for $(U, V) \in W^{s}\left(M_{1}, 0\right) \backslash\left\{\left(M_{1}, 0\right)\right\}$. Since $N_{1}$ is bounded, the result follows.

LEMMA 4.2. Let $p$ be a hyperbolic rest point in a local flow on some manifold, and let $N$ be a compact neighborhood of $p$. Suppose that each $x \in$ $\left(W^{u}(p) \cup W^{s}(p)\right) \backslash\{p\}$ leaves $N$ in either forward or backward time. Then there is a neighborhood $U$ of $p$ such that each point of $U \backslash\{p\}$ leaves $N$ in either forward or backward time.

ProOF. By Hartman's theorem, there is a neighborhood $V$ of $p$ such that the flow in $V$ in conjugate to the linearized flow in $V$. By the basic theory of linear equations, there are coordinates $\left(x_{1}, \ldots, x_{d}\right)$ in some neighborhood of $p$ with the origin at $p$ and such that the flow has the form $(x, t) \rightarrow\left(e^{t} x_{1}, \ldots, e^{t} x_{k}, e^{-t} x_{k+1}, \ldots, e^{-t} x_{d}\right)$. If $k=0$ or $k=d$, then the result is clear. Otherwise, choose $\varepsilon$ such that $\left\{\left(x_{1}, \ldots, x_{d}\right)|| x_{i} \mid<2 \varepsilon\right.$ for each $\left.i\right\} \subset V$. Note that locally, $W^{u}(p)=\left\{\left(x_{1}, \ldots, x_{d}\right) \mid\right.$ $x_{i}=0$ for $\left.i=k+1, \ldots, d\right\}, W^{s}(p)=\left\{\left(x_{1}, \ldots, x_{d}\right) \mid x_{i}=0\right.$ for $\left.i=1, \ldots, k\right\}$.

Let $K_{1}=\left\{\left(x_{1}, \ldots, x_{k}, 0, \ldots, 0\right)|| x_{i} \mid \leq \varepsilon\right.$ for $i=1, \ldots, k$ and $x_{j}=\varepsilon$ for some $\left.j\right\}$. Then $K_{1}$ is a topological $(d-1)$-sphere and $K_{1} \subset W^{u}(p)$ so each point leaves $N$ in forward time. For $0<\delta<\varepsilon$ let

$$
\begin{aligned}
K^{\delta}=\left\{\left(x_{1}, \ldots, x_{d}\right)|| x_{i} \mid \leq \varepsilon \text { for } i \leq k,\left|x_{l}\right|=\right. & \varepsilon \text { for some } l \leq k \\
& \text { and } \left.\left|x_{j}\right| \leq \delta \text { for } j=k+1, \ldots, d\right\} .
\end{aligned}
$$

Since $N$ is closed and $K_{1}$ is compact, each point in $K^{\delta}$ leaves $N$ in forward time if $\delta$ is small enough. Choose such a $\delta$ and let

$$
U=\left\{\left(x_{1}, \ldots, x_{d}\right)|| x_{i} \mid \leq \varepsilon \text { for } i \leq k,\left|x_{j}\right| \leq \delta \text { for } j=k+1, \ldots, d\right\} .
$$

Then $U$ is a neighborhood of $p$. If $x \in U \backslash W^{s}(p)$, the $x$ will intersect $K^{\delta}$ in forward time, so $x$ leaves $N$ in forward time. If $x \in W^{s}(p) \backslash\{p\}$, then $x$ leaves in backward time by hypothesis.

Lemmas 4.1 and 4.2 show that $\mathrm{A} 4$ implies $\mathrm{A} 4^{\prime}$, so Theorem 1.5 is proved. In the course of proving this we showed that $\mathrm{A} 4$ can be replaced by the conclusion of Lemma1 4.1, i.e. that every point in the stable and unstable manifold of $\left(M_{1}, 0\right)$ except the rest point itself leave $N_{1} \times \mathbf{R}^{d}$ in forward or backward time. 
Degree theory and the deformation $H_{1}$ show that the function $\left(2 F\left(M_{1}\right)-2 F(U)-\right.$ $\langle U, \nabla F(U)\rangle)$ could have just one zero in $N_{1}$, namely $M_{1} . M_{1}$ is a minimum, so the positivity is assured if $M_{1}$ is the only zero in $N_{1}$.

\section{REFERENCES}

1. C. C. Conley, Isolated invariant sets and the Morse index, CBMS Regional Conf. Ser. in Math., vol. 38, Amer. Math. Soc., Providence, R.I., 1978.

2. R. Franzosa, Index filtrations and connection matrices for partially ordered Morse decompositions, Thesis, Univ. of Wisconsin, Madison, 1984.

3. _ Index filtrations and the homology index braid for partially ordered Morse decompositions, Trans. Amer. Math. Soc. 298 (1986), 193-213.

4. _ , The connection matrix theory for Morse decompositions, Trans. Amer. Math. Soc. (to appear).

5. J. Reineck, Connecting orbits in one parameter families of flows, J. Ergodic Theory Dynamical Systems (to appear).

6. D. Salamon, Connected simple systems and the Conley index of isolated invariant sets, Trans. Amer. Math. Soc. 29 (1985), 1-41.

7. D. Terman, Infinitely many travelling wave solutions of a gradient system, preprint.

DEPARTMENT OF MATHEMATICS, NORTHWESTERN UNIVERSITy, EVANSTON, ILLINOIS 60201

Current address: Department of Mathematics State University of New York at Buffalo, Buffalo, New York 14214 